Ocular Oncology

and Pathology
Ocul Oncol Pathol 2017;3:11-16

DOI: $10.1159 / 000448144$
Received: June 2, 2016

Accepted: July 1, 2016

Published online: September 7, 2016

\title{
Congenital Orbital Teratoma
}

\author{
Fernando Pellerano ${ }^{a}$ b Elvis Guillermo ${ }^{c}$ Gloreley Garrido ${ }^{c}$ Pedro Berges ${ }^{d}$ \\ ${ }^{a}$ Ocular Diagnostic Unit, Clínica Oftalmológica de Santo Domingo, b Universidad Iberoamericana (UNIBE) School of \\ Medicine, and Departments of ${ }^{c}$ Pediatric Ophthalmology and d Pathology, Hospital Infantil Dr. Robert Reid Cabral, \\ Santo Domingo, Dominican Republic
}

\section{Key Words}

Congenital teratoma · Orbital tumor · Proptosis in a neonate

\begin{abstract}
We report a case of congenital orbital teratoma. A 3-day-old male, born at 39 weeks' gestation without relevant prenatal history, presented with a large vascularized proptotic mass distorting the left midface. Laboratory studies showed elevated serum alpha-fetoprotein (12,910 ng/ml). Computed tomography showed a multiloculated heterogeneous lesion composed of hypodense and hyperdense calcified areas encompassing the whole orbital cavity with expansion of the bony walls, as well as forward displacement and compression of the eyeball without extension to surrounding structures. Clinical, imaging and laboratory features were consistent with congenital orbital teratoma. Due to pronounced proptosis with exposure keratopathy and corneal perforation, no motility of the globe and no vision in the affected eye in a resource-limited setting, the patient underwent orbital exenteration. Histopathological examination confirmed the diagnosis of mature cystic teratoma. We describe the clinical course, radiographic and histopathological findings of this rare orbital tumor.
\end{abstract}

(c) 2016 S. Karger AG, Basel

\section{KARGER}

(c) 2016 S. Karger AG, Basel

E-Mail karger@karger.com

www.karger.com/oop

\section{Introduction}

Teratomas are tumors composed of tissues derived from all three germ layers [1]. Orbital location is rare $(0.8 \%)$ [2] and usually occurs in the left orbit of healthy newborns, with a female:male ratio of 2:1 [3]. Clinically, the tumors present with rapid growth, unilateral proptosis and palpebral retraction without intracranial involvement [4]. They may enlarge the orbit to three times its normal size [5]. At imaging, benign orbital teratomas are usually multiloculated, cystic masses with an admixture of tissues including calcification, fat and ossification. Most orbital teratomas are sharply circumscribed, benign and well differentiated histologically [6]. To our knowledge, this is the first reported case in the Dominican Republic and the Caribbean. Due to its extreme rarity, we herein describe the clinical course, radiographic and histopathological findings of this case.

\section{Case Report}

A 3-day-old male, born at 39 weeks' gestation without relevant prenatal history, presented to the ophthalmology service with a large vascularized proptotic mass distorting the left midface. No intrauterine ultrasound had been performed and delivery had been unremarkable. Examination showed eyelid retraction with chemo- 

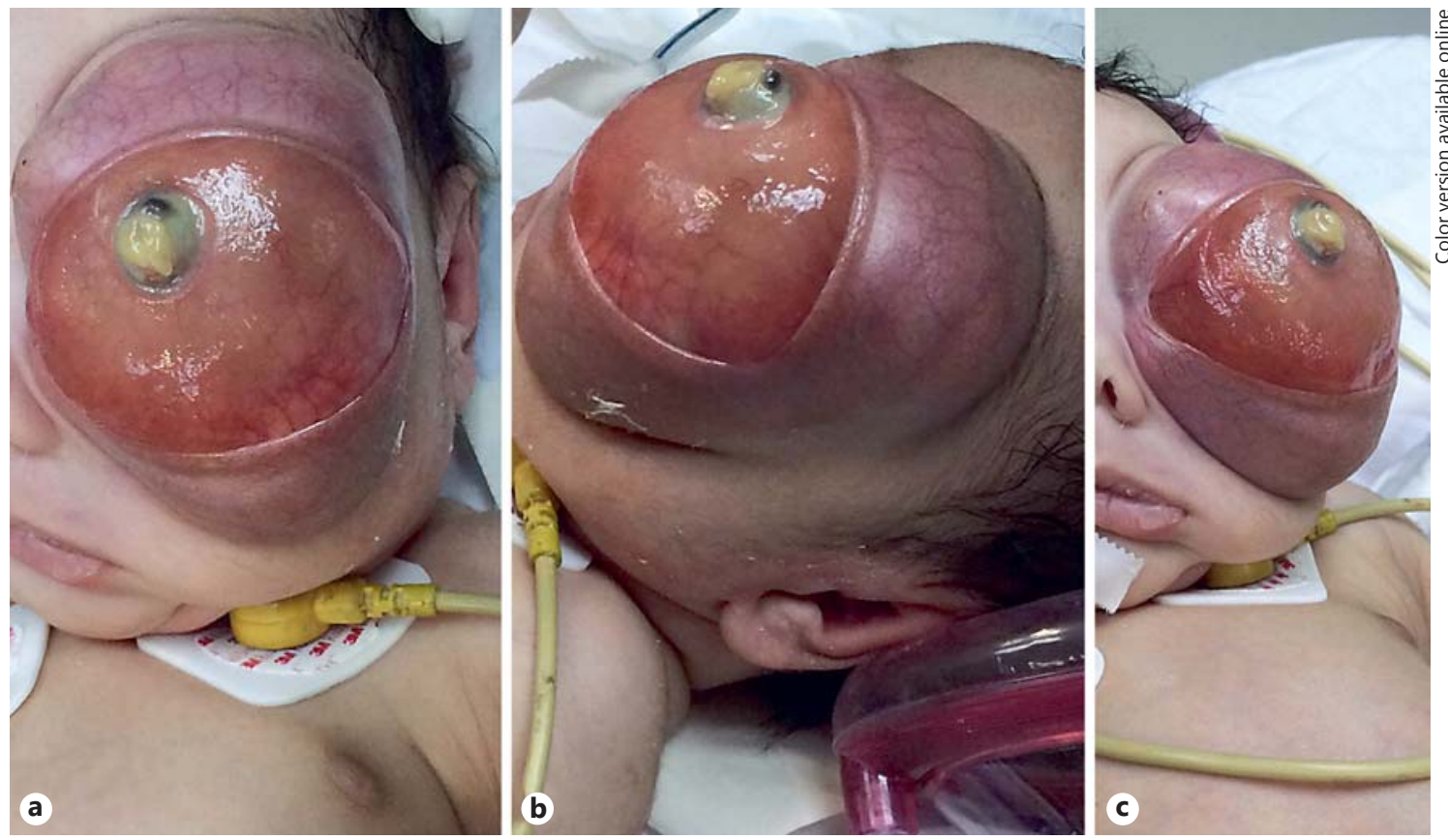

Fig. 1. a, b Frontal (a) and lateral (b) preoperative images of the left orbital mass showing eyelid retraction with chemosis, exposure keratopathy with corneal perforation, and anterior chamber inflammation with hypopyon. c Left nostril compression and downward displacement of the left angle of the mouth.

sis, exposure keratopathy with corneal perforation, and anterior chamber inflammation with hypopyon (fig. 1a, b). The mass was nonpulsatile with areas of transillumination, producing left nostril compression and downward displacement of the left angle of the mouth (fig. 1c). There was no motility of the globe and no view of the fundus in the affected eye. The right eye was normal, with no other findings. Laboratory studies showed an elevated serum alpha-fetoprotein $(12,910 \mathrm{ng} / \mathrm{ml})$. Computed tomography (CT) scans showed a multiloculated heterogeneous lesion composed of hypodense and hyperdense calcified areas encompassing the whole orbital cavity with expansion of the bony walls (fig. $2 a, b$ ) as well as forward displacement and compression of the eyeball (fig. 2c) without extension to surrounding structures. The extraocular muscles and optic nerve could not be identified.

Clinical, imaging and laboratory features were consistent with congenital orbital teratoma. Due to pronounced proptosis with exposure keratopathy and corneal perforation, no motility of the globe and no vision in the affected eye in a resource-limited setting, the patient underwent orbital exenteration with no intraoperative complications. Postoperatively he was given analgesics and antibiotics, and the empty orbit was managed with daily saline and white vinegar to promote wound healing.

Macroscopically, the tumor was ovoid with a hard-elastic consistency, measuring $7.0 \times 5.5 \times 4.5 \mathrm{~cm}$ (fig. $3 \mathrm{a}$ ). Serial sections revealed cystic cavities containing clear fluid and tissue with gray-red areas of hard consistency resembling bone and cartilage. Histological sections exhibited a benign neoplasm of ectodermal, mesodermal and endodermal origin including neuroepithelium, keratinized stratified squamous epithelium with appendages (fig. 3b), sebaceous glands, bone and cartilage (fig. 3c), adipose tissue, skeletal muscle, blood vessels, bone marrow and ciliated pseudostratified columnar epithelium with goblet cells resembling bronchial epithelium (fig. 3d). No immature or malignant tissue was identified. Histopathological examination confirmed the diagnosis of mature cystic teratoma.

The patient was discharged on the 14th postoperative day without any complications and with normal levels of serum alpha-fetoprotein. Two months (fig. 4a) and 12 months (fig. 4b) after surgery he was stable with no recurrence of the tumor. A customized orbital prosthesis may be used in the future.

\section{Discussion}

Orbital teratomas are rare, usually benign congenital tumors [6], with about 70 cases reported in the English language literature, including two cases from India [7]. Orbital teratomas have been divided into four groups: (1) complete orbital fetus-in-fetus - orbitopagus parasiticus, (2) a portion of a second fetus in the orbit, (3) true orbital teratoma with all three germ layers, and (4) a tumor with only two germ cell lines [8]. True orbital teratomas, as in our case, are rare. Spinelli et al. [9] in 1993 reviewed only 51 well-documented patients with true congenital orbital teratomas. 

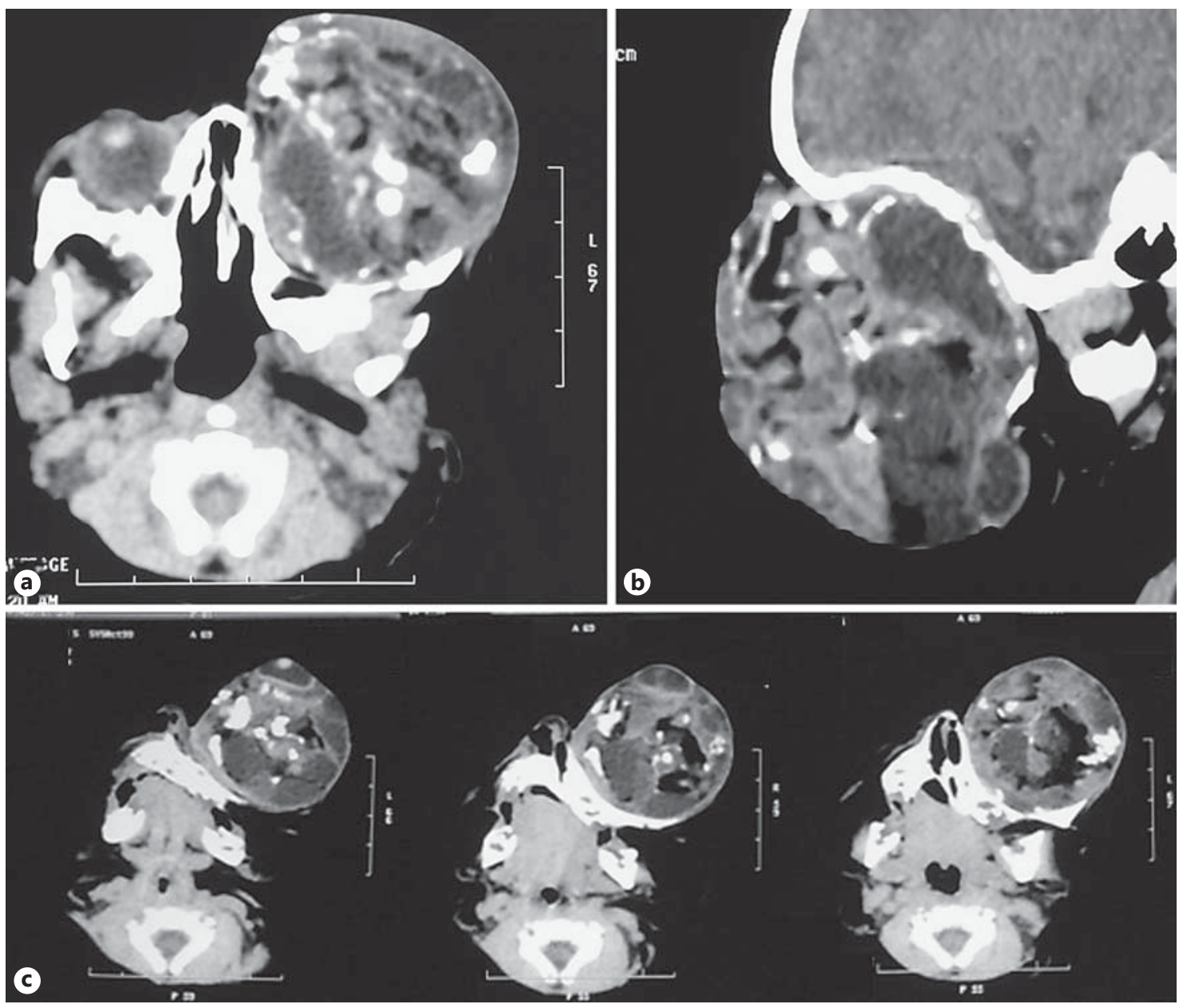

Fig. 2. a, b Axial (a) and sagittal (b) CT scans showing a heterogeneous lesion composed of hypodense and hyperdense calcified areas encompassing the whole orbital cavity with expansion of the bony walls. c Multiple axial cuts showing forward displacement and compression of the eyeball.

Clinically, the tumor presents in healthy newborns with rapid growth, extreme unilateral proptosis and marked stretching of the eyelids over a tense, fluctuating mass, with elongation of the palpebral fissure and transillumination of all or part of the orbital mass [5]. The persistent enlargement of this neoplasm is attributed to mucus secretion from the embryonic intestinal tissue [10]. The eye is usually normally formed, but displaced superiorly and forward. In rare cases there is no organized eye, with only remnants of ocular tissues being present [11]. The optic nerve may be encased or adherent to the tumor, leading to secondary atrophy and poor pupil reaction. Other features include no family history of congenital deformities with nonconsanguineous parents, normal pregnancy and delivery, and no history of teratogenic influences to the mother [12].
At imaging, benign orbital teratomas are usually multiloculated, cystic masses with an admixture of tissues including calcification, fat and ossification [6]. As in our case, CT imaging shows orbital enlargement with focal calcification in the lumen of the tumor. Erosion of the greater wing of the sphenoid may be seen [10].

The differential diagnosis of orbital teratoma includes microphthalmos with cyst, dermoid cyst, epidermoid inclusion cysts, hemangioma, lymphangioma, cephalocele, neuroblastoma, rhabdomyosarcoma and retinoblastoma $[6,13]$. Microphthalmos with cyst is distinguished on the basis of a small globe in conjunction with a communicating channel between it and an attached cyst [14]. Dermoid cysts are unilocular and have a single fat-fluid level. Epidermoid inclusion cysts, probably because of their proteinaceous contents, are usually similar in appearance

Ocul Oncol Pathol 2017;3:11-16 

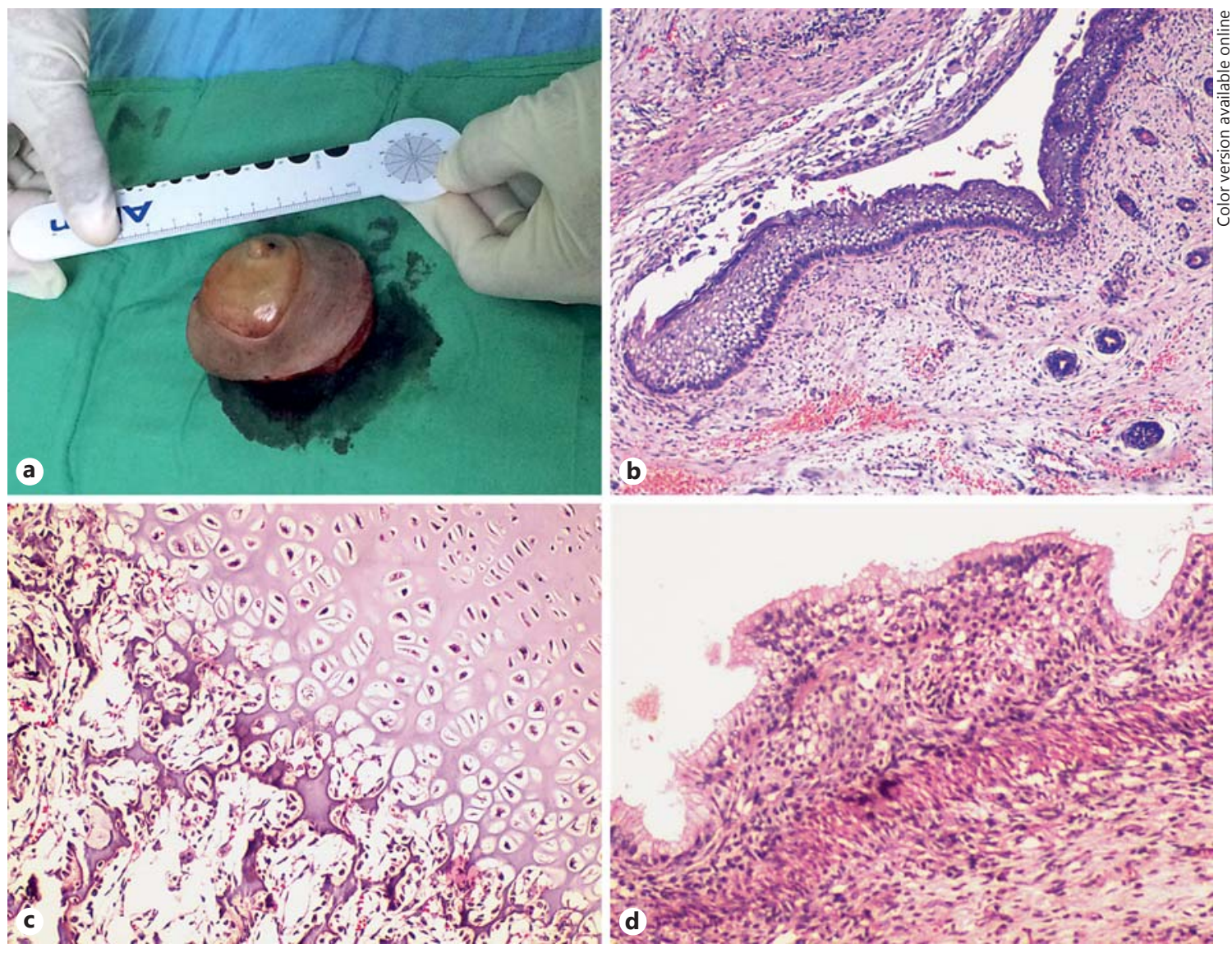

Fig. 3. a Macroscopic appearance of the resected specimen. b Low-magnification image of ectodermal component: keratinized stratified squamous epithelium with appendages. c High-magnification image of mesodermal component: bone and cartilage. $\mathbf{d}$ Low-magnification image of endodermal component: ciliated pseudostratified columnar epithelium with goblet cells resembling bronchial epithelium. b-d Hematoxylin and eosin: $\times 4($ b $), \times 40$ (c) and $\times 10(\mathbf{d})$.

to water or cerebral spinal fluid on CT. Lymphangiomas are multilocular, but do not contain fat or calcification. Cephaloceles contain cerebral spinal fluid and generally have adjacent osseous abnormalities, but unlike teratomas and dermoids, cephaloceles do not contain lipid [6]. Rhabdomyosarcoma, neuroblastoma and retinoblastoma should be considered in children presenting after the newborn period. The first two show evidence of bone destruction and the latter intraocular mass lesion or calcification [13].

Surgical excision is the treatment of choice [15]. Although preservation of the globe has been successfully performed in some cases of teratoma $[16,17]$, the tumor may grow rapidly, causing destructive proptosis and exposure keratopathy within days, with resultant poor prognosis for vision or conservation of the globe [18]. The reasons stated above and the resource-limited environments of public hospitals in the Dominican Republic were the deciding factors to perform orbital exenteration in this patient.

The predominant germ cell observed in orbital teratomas is surface ectoderm as cysts filled with keratin and adnexal structures, such as hair follicles and sweat glands. Neuroectodermal tissues include primitive neural tubes, choroidal plexus, ganglia, and glial elements. The mesoderm is the second most common germ cell layer, represented by muscle, bone, cartilage, and fat. The endoderm is the least common component of teratomas and may produce respiratory or gastrointestinal tissue cysts [12]. The definitive diagnosis of an orbital teratoma is made by 
Fig. 4. Two months (a) and 12 months (b) after the procedure.
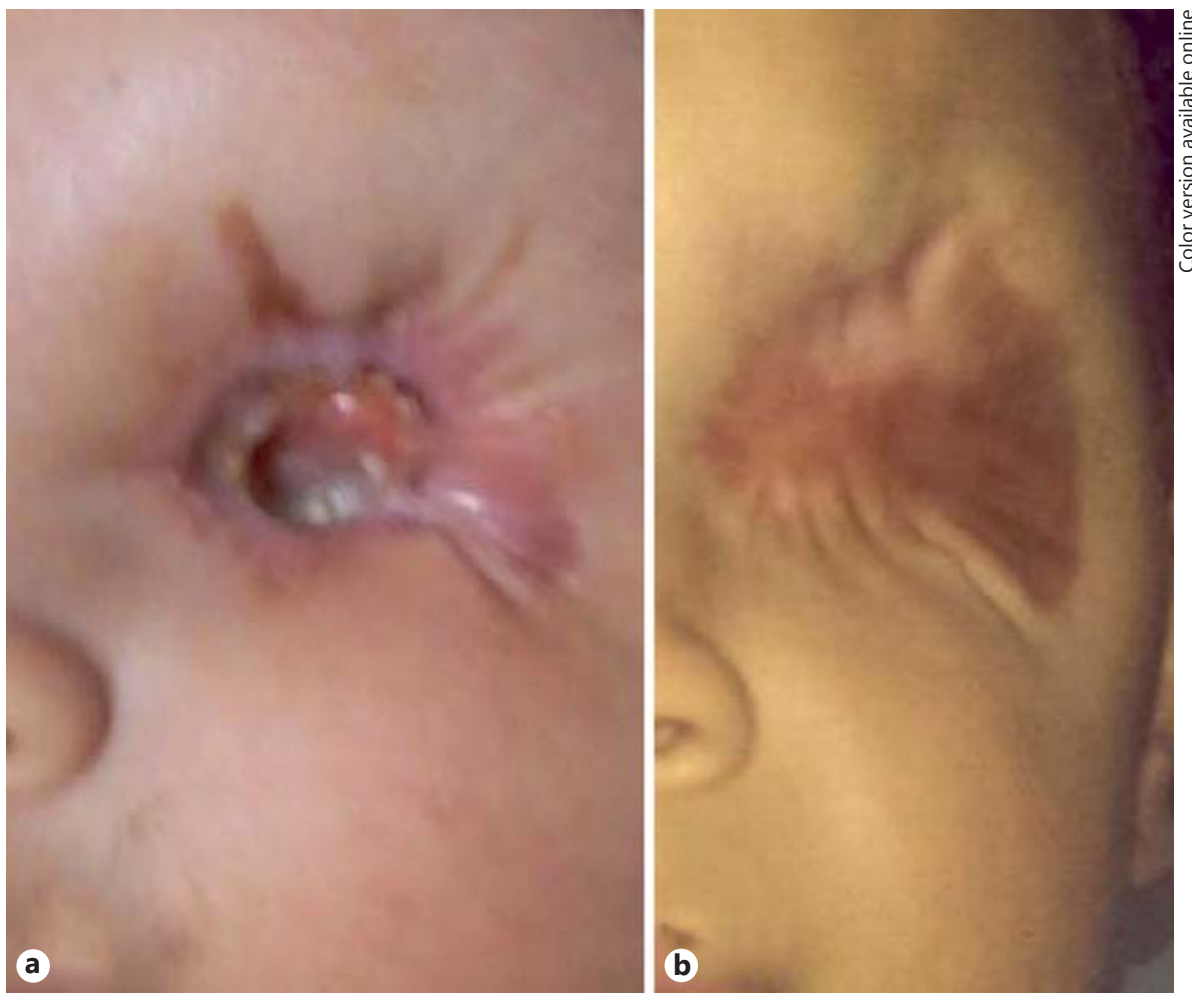

histopathology, which shows gut-like structures, the sine qua non for diagnosis of a teratoma [10].

Orbital teratomas have been known to recur and may undergo malignant degeneration. Therefore, close follow-up is necessary [10]. There is controversy on the diagnostic value of serum alpha-fetoprotein for mature teratomas [19], especially in the newborn period, being normally high as a result of fetal production [20]. However, elevated serum alpha-fetoprotein levels may signal the presence of regional recurrence or metastatic disease in the setting of malignant teratoma [21]. The levels in our patient were elevated preoperatively and returned back to normal postoperatively, with no recurrence of the tumor. Most congenital orbital teratomas have been shown to be largely benign in biologic behavior and are now being managed more conservatively with good cosmetic results and a better prognosis for vision [18].

This brief report adds to the body of world literature about true congenital orbital teratomas. In conclusion, congenital orbital teratomas are rare tumors that should be included in the differential diagnosis of proptosis in a neonate in order to ensure prompt management with good ocular prognosis and orbitofacial development.

\section{Statement of Ethics}

The study protocol was approved by the Committee on Human Research of Hospital Infantil Dr. Robert Reid Cabral and by the Ethics Committee of Universidad Iberoamericana. Informed consent was signed by the patient's mother.

\section{Disclosure Statement}

The authors declare no conflicts of interest.

References $\quad 1 \begin{aligned} & \text { Tapper D, Lack EE: Teratomas in infancy and } \\ & \text { childhood. A 54-year experience at the Chil- } \\ & \text { dren’s Hospital Medical Center. Ann Surg }\end{aligned}$
$1983 ; 198: 398-410$.


5 Gnanaraj L, Skibell BC, Coret-Simon J, Halliday W, Forrest C, DeAngelis DD: Massive congenital orbital teratoma. Ophthal Plast Reconstr Surg 2005;21:445-447.

6 Herman TE, Vachharajani A, Siegel MJ: Massive congenital orbital teratoma. J Perinatol 2009;29:396-397.

7 Sharma MC, Sarkar C, Gaikwad S, Mahapatra AK, Bahadur S: Congenital orbital teratoma: a report of two cases. Indian J Ophthalmol 1997;45:49-52.

8 Damato PJ, Damato FJ: Neonatal orbital teratoma. Br J Ophthalmol 1962;46:685-691.

9 Spinelli HM, Criscuolo GR, Tripps M, Buckley PJ: Massive orbital teratoma in the newborn. Ann Plast Surg 1993;31:453-458.

10 Patel B: Benign orbital tumors: teratoma; in Perry J, Singh A (eds): Clinical Ophthalmic Oncology: Orbital Tumors, vol 1. Berlin/Heidelberg/New York/Dordrecht/London, Springer, 2014, pp 83-84.
11 Sreenan C, Johnson R, Russel L, Bhargava R, Osiovich H: Congenital orbital teratoma. Am J Perinatol 1999;16:251-255.

12 González C, Restrepo C, Salazar G, Monsalve P: Congenital orbital teratoma: case report. Colomb Med (Cali) 2012;43:82-85.

13 Hann L, Borden S, Weber A: Orbital teratoma in the newborn. A case report. Pediatr Radiol 1977;5:172-174.

14 Singh U, Subramanian A, Bal A: Congenital orbital teratoma presenting as microphthalmos with cyst. Indian J Ophthalmol 2009;57: 474-475.

15 Fabian ID, Priel A, Fridman E, Rosen N, Barshak I, Greenberg G, Rosner M: Congenital orbital teratoma. Harefuah 2013;152:323$325,369$.

16 Mee JJ, McKelvie PA, McNab AA: Orbital teratoma: late presentation with normal vision. Clin Experiment Ophthalmol 2002;30:41-43.

17 Shields JA, Shields CL: Orbital cysts of childhood - classification, clinical features, and management. Surv Ophthalmol 2004;49:281299.
18 Ogun OA, Ogun GO, Brown BJ, Mosuro AL, Ashaye AO: Congenital orbital teratoma: a case report and challenges of its management in a resource limited setting. Pan Afr Med J 2012;12:3.

19 Tsuchida Y, Hasegawa H: The diagnostic value of alpha-fetoprotein in infants and children with teratomas: a questionnaire survey in Japan. J Pediatr Surg 1983;18:152-155.

20 Heerema-McKenney A, Bowen J, Hill A, Suster S, Qualman S: Protocol for the examination of specimens from pediatric and adult patients with extragonadal germ cell tumors. Arch Pathol Lab Med 2011;135:630-639.

21 Brodsky J, Kanwar V, Stafford L, Rahbar R, Frazier L: Germ cell tumors/teratoma; in Rahbar R, Rodriguez-Galindo C, Meara J, Smith E, Perez-Atayde (eds): Pediatric Head and Neck Tumors: A-Z Guide to Presentation and Multimodality Management, vol 1. New York, Springer Science+Business Media, 2014, p 155. 